\title{
Dimension-free Wasserstein contraction of nonlinear filters
}

\author{
Nick Whiteley \\ Institute for Statistical Science, School of Mathematics, University of Bristol \\ and the Alan Turing Institute
}

January 20, 2021

\begin{abstract}
For a class of partially observed diffusions, conditions are given for the map from the initial condition of the signal to filtering distribution to be contractive with respect to Wasserstein distances, with rate which does not necessarily depend on the dimension of the state-space. The main assumptions are that the signal has affine drift and constant diffusion coefficient and that the likelihood functions are log-concave. Ergodic and nonergodic signals are handled in a single framework. Examples include linear-Gaussian, stochastic volatility, neural spike-train and dynamic generalized linear models. For these examples filter stability can be established without any assumptions on the observations.
\end{abstract}

\section{Introduction}

\subsection{Setting}

Let $\left(X_{t}\right)_{t \in \mathbb{R}_{+}}$, called the signal process, be the solution of the stochastic differential equation:

$$
\mathrm{d} X_{t}=\left(\alpha+\beta X_{t}\right) \mathrm{d} t+\sigma \mathrm{d} B_{t},
$$

where $\alpha \in \mathbb{R}^{p}$ and $\beta$ is a $p \times p$ matrix of reals, $\sigma \geq 0$ is a scalar, and $\left(B_{t}\right)_{t \in \mathbb{R}_{+}}$is $p$-dimensional Brownian motion. Let observations $\left(Y_{k}\right)_{k \in \mathbb{N}_{0}}$ be each valued in a measurable space $(\mathbb{Y}, \mathcal{Y})$, conditionally independent given $\left(X_{t}\right)_{t \in \mathbb{R}_{+}}$, and such that the conditional probability that $Y_{k}$ lies in $A \in \mathcal{Y}$ given $\left(X_{t}\right)_{t \in \mathbb{R}_{+}}$is of the form $\int_{A} g_{k}\left(X_{k \Delta}, y\right) \chi(\mathrm{d} y)$, for a measure $\chi$ on $\mathcal{Y}$, a function $g_{k}: \mathbb{R}^{p} \times \mathbb{Y} \rightarrow(0, \infty)$ and a constant $\Delta>0$.

The filtering distributions $\pi_{k}\left(x, y_{0: k}, \cdot\right), k \in \mathbb{N}_{0}$, on the Borel sigma algebra $\mathcal{B}\left(\mathbb{R}^{p}\right)$, associated with an initial state $x$ and a realized observation sequence $\left(y_{k}\right)_{k \in \mathbb{N}_{0}}$, are defined by

$$
\pi_{k}\left(x, y_{0: k}, A\right):=\frac{\mathbf{E}_{x}\left[\mathbf{1}_{A}\left(X_{k \Delta}\right) \prod_{j=0}^{k} g_{j}\left(X_{j \Delta}, y_{j}\right)\right]}{\mathbf{E}_{x}\left[\prod_{j=0}^{k} g_{j}\left(X_{j \Delta}, y_{j}\right)\right]}, \quad A \in \mathcal{B}\left(\mathbb{R}^{p}\right),
$$

where $\mathbf{E}_{x}$ denotes expectation with respect to the law of the solution of (1.1) with $X_{0}=x$. When $\left(y_{0}, \ldots, y_{k}\right)$ are replaced in $(1.2)$ by the random variables $\left(Y_{0}, \ldots, Y_{k}\right)$ distributed according to the above prescription and with true initialization also $X_{0}=x$, then $\pi_{k}\left(x, y_{0: k}, \cdot\right)$ is a version of the conditional distribution of $X_{k \Delta}$ given $\left(Y_{0}, \ldots, Y_{k}\right)$. It shall be assumed throughout that whichever $x$ and $\left(y_{k}\right)_{k \in \mathbb{N}_{0}}$ we consider, the denominator in (1.2) is finite for each $k$, which combined with $g_{k}(x, y)>0$ implies that $\pi_{k}\left(x, y_{0: k}, \cdot\right)$ is well defined as a probability measure.

The filtering problem - computing or approximating the distributions (1.2) - appears across Bayesian statistics, machine learning and signal processing [12, 18, 9] and a broard literature on 
its mathematical analysis has developed [3]. The question of under what conditions the filtering distributions are stable with respect to their initial condition has a rich history and has been addressed using a wide variety of techniques, an overview of the field is given in [3, Chap. 4].

Relatively recent results from $[15,7,8,11]$ are applicable to the model class described above, or some instances thereof, under appropriate technical conditions. They establish quantitative bounds on the total variation distance, or a weighted version thereof in [11], between differently initialized filtering distributions and obtain rate estimates which depend on constants associated with minorization-type conditions for the signal process. However such constants, and therefore the rate estimates obtained from them, typically degrade with the dimension of the state-space. The emphasis of the present work is on identifying techniques and assumptions which allow this issue to be overcome.

Also recently, infinite-dimensional filtering has been treated in [27], where stability results are obtained involving weak convergence and the notion of local ergodicity, which pertains to the mixing properties of non-Markovian, finite-dimensional components of an infinite dimensional signal process, conditional on the observations. The results hold under very mild conditions which cannot be expected to yield a particular rate of convergence. As part of a study of particle filters for signals with certain spatio-temporal mixing properties, [22] uses the Dobrushin comparison theorem to obtain quantitative filter stability results with respect to local variation norms, which do not degrade with dimension.

\subsection{Outline of the approach}

The approach taken here does not rely on spatial structure of the model, but is instead connected with contraction properties of gradient flows and convexity, and influenced by analyses of Markov processes using abstract ideas of curvature and underlying links to functional inequalities [1, 2]. The proofs ultimately rely on a quite simple coupling technique and the pathwise stability properties of diffusions whose drifts involve the gradients of certain convex potentials. This convexity arises from a combination of two features of the model we consider: firstly a log-concavity-preservation characteristic of the signal model (1.1), and secondly log-concavity of the likelihood functions $x \mapsto g_{k}(x, y)$ (precise assumptions are stated later).

Regarding the first feature, it is known that the transition kernels $\left(P_{t}\right)_{t \in \mathbb{R}_{+}}$associated with (1.1) preserve log-concavity, meaning that for any log-concave function $f$ and $t>0, P_{t} f$ is log-concave, see for example [16]. If for each $k$ and $y$ the likelihood function $x \mapsto g_{k}(x, y)$ is log-concave, then the Markov property of $\left(X_{t}\right)_{t \in \mathbb{R}_{+}}$and the fact that a pointwise product of log-concave functions is

log-concave imply that the function $x \mapsto \mathbf{E}_{x}\left[\prod_{i=j}^{k} g_{i}\left(X_{(i-j) \Delta}, y_{i}\right)\right]$ is log-concave for any $y_{j}, \ldots, y_{k}$. Functions of this form play an important role in filter stability because they provide the re-weighting of transition probabilities which corresponds to conditioning on observations, and this is where the convex potentials alluded to earlier arise.

It is important to note that log-concavity of $x \mapsto \mathbf{E}_{x}\left[\prod_{i=j}^{k} g_{i}\left(X_{(i-j) \Delta}, y_{i}\right)\right]$ cannot be expected in much greater generality. It was established in [16] that among all diffusions of the form:

$$
\mathrm{d} X_{t}=b\left(X_{t}\right) \mathrm{d} t+\sigma\left(X_{t}\right) \mathrm{d} B_{t},
$$

with $b(\cdot), \sigma(\cdot)$ satisfying some mild regularity conditions, it is only in the case that $b(\cdot)$ is affine and $\sigma(\cdot)$ is a constant that $P_{t} f$ is log-concave for all log-concave $f$. This motivates our focus on signal processes of the form (1.1).

Having emphasized the central role of convexity in the present work, let us finally note the results presented here complement those of [25], who studied filter stability for a class of diffusions which are linearly observed in continuous time:

$$
\begin{aligned}
\mathrm{d} X_{t} & =\beta X_{t} \mathrm{~d} t+C C^{T} \nabla \log \phi\left(X_{t}\right) \mathrm{d} t+C \mathrm{~d} B_{t}, \\
\mathrm{~d} Y_{t} & =G X_{t} \mathrm{~d} t+\Gamma \mathrm{d} W_{t},
\end{aligned}
$$


where $\beta, C, G, \Gamma$ are matrices of appropriate size, $W_{t}$ is $p$-dimensional Brownian motion and $R:=\Gamma \Gamma^{T}$ is invertible. Stability is proved in [25] via certain diffusion contraction estimates with respect to Lipschitz norms, under the condition that

$$
x \mapsto V(x):=\langle\beta x, \nabla \log \phi(x)\rangle+\frac{1}{2} \frac{\operatorname{tr}\left(Q \phi^{\prime \prime}(x)\right)}{\phi(x)}+\frac{1}{2}\left\langle R^{-1} G x, G x\right\rangle
$$

is uniformly strictly convex. A rate of convergence in total variation distance is obtained in terms of the spectrum of the solution of a particular matrix Riccati equation. The setup (1.3)-(1.4) is a counterpart to the one considered in the present paper: in (1.3)-(1.4) the linearity is in the observation model, where as in (1.1) the linearity is in the signal and our discrete-time observations $\left(Y_{k}\right)_{k \in \mathbb{N}_{0}}$ may be related to the signal in a nonlinear way.

\subsection{Notation and conventions}

The Euclidean norm and inner-product on $\mathbb{R}^{p}$ are denoted $\|\cdot\|$ and $\langle\cdot, \cdot\rangle$. A function $f: \mathbb{R}^{p} \rightarrow(0, \infty)$ is called log-concave if

$$
\log f(c u+(1-c) v) \geq c \log f(u)+(1-c) \log f(v), \quad \forall u, v \in \mathbb{R}^{p}, c \in[0,1],
$$

and strongly log-concave if there exists a log-concave function $\tilde{f}$ and a constant $\lambda_{f} \in(0, \infty)$ such that $f(u)=\exp \left(-\frac{\lambda_{f}}{2}\|u\|^{2}\right) \tilde{f}(u)$. For a measure $\mu$, function $f$ and integral kernel $K$, we shall write $\mu f=\int f(u) \mu(\mathrm{d} u), \mu K(\cdot)=\int \mu(\mathrm{d} u) K(u, \cdot), K f(u)=\int f(v) K(u, \mathrm{~d} v)$. For a nonnegative function $f, \mu \cdot f$ denotes the measure $\mu(\mathrm{d} u) f(u)$. The gradient and Laplace operators with respect to $x$ are denoted $\nabla_{x}$ and $\nabla_{x}^{2}$. The indicator function on a set $A$ is denoted $\mathbf{1}_{A}$. The class of real-valued and twice continuously differentiable functions on $\mathbb{R}^{p}$ is denoted $C^{2}$.

The order- $q$ Wasserstein distance between probability measures on $\mathcal{B}\left(\mathbb{R}^{p}\right)$ is:

$$
W_{q}(\mu, \nu):=\left(\inf _{\gamma \in \Gamma(\mu, \nu)} \int_{\mathbb{R}^{p} \times \mathbb{R}^{p}}\|u-v\|^{q} \gamma(\mathrm{d} u, \mathrm{~d} v)\right)^{1 / q}
$$

where $\Gamma(\mu, \nu)$ is the set of all couplings of $\mu$ and $\nu$.

\section{Wasserstein distance between filtering distributions initial- ized at points}

\section{$2.1 \quad$ Main result}

Assumption 1. For each $k \in \mathbb{N}_{0}$ and $y \in \mathbb{Y}, x \mapsto g_{k}(x, y)$ is strictly positive, a member of $C^{2}$, and there exists $\lambda_{g}(k, y) \in[0, \infty)$ and a log-concave function $\tilde{g}_{k}(\cdot, y): \mathbb{R}^{p} \rightarrow(0, \infty)$ such that $g_{k}(x, y)=$ $\exp \left[-\frac{\lambda_{g}(k, y)}{2}\|x\|^{2}\right] \tilde{g}_{k}(x, y)$.

Theorem 1. If assumption 1 holds, then for any $q \geq 1, k \geq 1$ and $y_{0}, \ldots, y_{k} \in \mathbb{Y}$,

$$
W_{q}\left(\pi_{k}\left(x, y_{0: k}, \cdot\right), \pi_{k}\left(x^{\prime}, y_{0: k}, \cdot\right)\right) \leq \exp \left[-\sum_{j=1}^{k} \int_{0}^{\Delta} \lambda\left(j, y_{j}, t\right) \mathrm{d} t\right]\left\|x-x^{\prime}\right\|, \quad \forall x, x^{\prime} \in \mathbb{R}^{p},
$$

where

$$
\lambda(j, y, t):=\lambda_{\mathrm{sig}}+\frac{\sigma^{2} \lambda_{g}(j, y) \lambda_{\beta}^{\min }(\Delta-t)}{1+\sigma^{2} \lambda_{g}(j, y) \int_{t}^{\Delta} \lambda_{\beta}^{\max }(\Delta-s) \mathrm{d} s},
$$

$\lambda_{\text {sig }} \in \mathbb{R}$ is the minimum eigenvalue of $-\left(\beta+\beta^{T}\right) / 2$ and $\lambda_{\beta}^{\min }(t), \lambda_{\beta}^{\max }(t) \in(0, \infty)$ are respectively the minimum and maximum eigenvalues of $e^{\beta t}\left(e^{\beta t}\right)^{T}$. 


\subsection{Proof of theorem 1}

Let $\left(y_{k}\right)_{k \in \mathbb{N}_{0}}$ be an arbitrary sequence in $\mathbb{Y}$. This sequence will remain fixed throughout sections 2.22.4. To avoid cumbersome formulae, the dependence of some quantities on this sequence $\left(y_{k}\right)_{k \in \mathbb{N}_{0}}$ will not be shown in the notation, but in some places will be commented on in the text for avoidance of doubt.

Fix $k \geq 0$ and define

$$
\begin{aligned}
& \varphi_{k, k}(x):=g_{k}\left(x, y_{k}\right), \\
& \varphi_{j, k}(x):=g_{j}\left(x, y_{j}\right) P_{\Delta} \varphi_{j+1, k}(x), \quad 0 \leq j<k, \\
& R_{j, k}(x, A):=\frac{\int_{A} P_{\Delta}\left(x, \mathrm{~d} x^{\prime}\right) \varphi_{j, k}\left(x^{\prime}\right)}{P_{\Delta} \varphi_{j, k}(x)}, \quad 1 \leq j \leq k .
\end{aligned}
$$

The dependence of $\varphi_{j, k}$ and $R_{j, k}$ on $y_{j}, \ldots, y_{k}$ is not shown in the notation. Here the presentation is heavily influenced by the semigroup formulation of [4].

We will need the following preliminary results concerning log-concave functions.

Lemma 1. For any given $f: \mathbb{R}^{p} \rightarrow(0, \infty)$ which is a member of $C^{1}$ and $\lambda_{f} \geq 0$, conditions 1)-3) are equivalent:

1) There exists a log-concave function $\tilde{f}$ such that $f(u)=\exp \left(-\frac{\lambda_{f}}{2}\|u\|^{2}\right) \tilde{f}(u), \forall u \in \mathbb{R}^{p}$.

2) $\log f(u) \leq \log f(v)+\langle\nabla \log f(v), u-v\rangle-\frac{\lambda_{f}}{2}\|u-v\|^{2}, \forall u, v \in \mathbb{R}^{p}$.

3) $\langle\nabla \log f(u)-\nabla \log f(v), u-v\rangle \leq-\lambda_{f}\|u-v\|^{2}, \forall u, v \in \mathbb{R}^{p}$.

Proof. These equivalences are immediate consequences of elementary properties of strongly convex $C^{1}$ functions, see for example [19, Sec 2.1.3].

Lemma 2. For every log-concave $f$ and $t>0, P_{t} f$ is log-concave.

Proof. [16, proof of Prop. 1.3]

Lemma 3. We have

$$
\pi_{k}\left(x, y_{0: k}, A\right)=R_{1, k} R_{2, k} \cdots R_{k, k}(x, A) .
$$

If assumption 1 holds, then for each $j, k$ such that $0 \leq j \leq k$, there exists a log-concave function $x \mapsto \tilde{\varphi}_{j, k}(x)$, depending on $y_{j}, \ldots, y_{k}$, such that:

$$
\varphi_{j, k}(x)=\exp \left[-\frac{\lambda_{g}\left(j, y_{j}\right)}{2}\|x\|^{2}\right] \tilde{\varphi}_{j, k}(x) .
$$

Proof. The expression for $\pi_{k}\left(x, y_{0: k}, A\right)$ follows from (1.2) and the Markov property of the signal, this key identify can be traced back to [5]. The second claim is established using assumption 1, repeated application to (2.3)-(2.4) of lemma 2 and the fact that the pointwise product of log-concave functions is log-concave.

The main steps in the proof of theorem 1 from hereon are:

1. Lemma 4 in section 2.3 establishes that each Markov kernel $R_{j, k}$ can be interpreted in terms of the transition probabilities of an $h$-transform of the signal process (2.3), where $h$ is function which depends on $y_{j}, \ldots, y_{k}$ via $\varphi_{j, k}$. This transformation amounts to the addition of an extra "drift" term to the extended space-time generator (defined below) associated with the signal, where the extra term depends on $h$. 
2. Proposition 1 in section 2.3 bounds the Wasserstein distance between $R_{j, k}(x, \cdot)$ and $R_{j, k}\left(x^{\prime}, \cdot\right)$ using a synchronous coupling of these $h$-transformed diffusions, assuming log-concavity of the $h$-function in its spatial argument. Specifically, in the proof of proposition 4 the Wasserstein distance is bounded in terms of the Euclidean distance between the paths of the coupled diffusions, which is in turn controlled by $\lambda_{\text {sig }}$ and the strength of the log-concavity of the $h$-function. Roughly speaking, the stronger this log-concavity is, the stronger the Wasserstein contraction of $R_{j, k}(x, \cdot)$ is.

3. Proposition 2 in section 2.4 establishes that the $h$-function is indeed log-concave in its spatial argument, and quantifies the strength of its log-concavity. In the proof of proposition 2 this log-concavity is inherited from that of $\varphi_{j, k}$ as per (2.6), and it is from here that the constants $\lambda_{g}\left(k, y_{k}\right)$ from assumption 1 appear in the log-concavity of $h$ and hence ultimately in the bounds of proposition 1.

4. Finally, the bound on $W_{q}\left(\pi_{k}\left(x, y_{0: k}, \cdot\right), \pi_{k}\left(x^{\prime}, y_{0: k}, \cdot\right)\right)$ given in theorem 1 is an immediate consequence of proposition 1 combined with (2.5).

\subsection{A space-time $h$-transform of the signal process}

Let $C\left([0, \Delta], \mathbb{R}^{p} \times[0, \Delta]\right)$ be the space of $\mathbb{R}^{p} \times[0, \Delta]$-valued, continuous functions on $[0, \Delta]$ endowed with the supremum norm. Let $\left(X_{t}, t\right)_{t \in[0, \Delta]}$ be the associated space-time coordinate process and let $\mathcal{F}=\left(\mathcal{F}_{t}\right)_{t \in[0, \Delta]}$ be the filtration it generates. The extended generator (in the sense of [23, p. 285]) of the space-time process on $C\left([0, \Delta], \mathbb{R}^{p} \times[0, \Delta]\right)$ under the law associated with (1.1) and acting on functions $f$ on $\mathbb{R}^{p} \times \mathbb{R}_{+}$is:

$$
L f(x, t):=\frac{\partial}{\partial t} f(x, t)+(\alpha+\beta x)^{T} \nabla_{x} f(x, t)+\frac{\sigma^{2}}{2} \nabla_{x}^{2} f(x, t) .
$$

Lemma 4. Let assumption 1 hold, fix any $j, k$ such that $1 \leq j \leq k$ and define

$$
h(x, t):=P_{\Delta-t} \varphi_{j, k}(x),
$$

where the dependence of $h$ on $j, k$ and $y_{j}, \ldots, y_{k}$ is not shown in the notation. There exists a probability kernel $\mathbf{P}^{h}: \mathbb{R}^{p} \times \mathcal{F}_{\Delta} \rightarrow[0,1]$ such that for any $x_{0} \in \mathbb{R}^{p}$ and $A \in \mathcal{B}\left(\mathbb{R}^{p}\right), R_{j, k}\left(x_{0}, A\right)=\mathbf{P}^{h}\left(x_{0},\left\{X_{\Delta} \in\right.\right.$ $A\})$, and under $\mathbf{P}^{h}\left(x_{0}, \cdot\right)$ the extended generator of the space-time process $\left(X_{t}, t\right)_{t \in[0, \Delta]}$ on $C\left([0, \Delta], \mathbb{R}^{p} \times\right.$ $[0, \Delta])$ is:

$$
L^{h} f(x, t):=L f(x, t)+\sigma^{2} \nabla_{x} \log h(x, t)^{T} \nabla_{x} f(x, t) .
$$

Proof. Let $\mathbf{P}: \mathbb{R}^{p} \times \mathcal{F}_{\Delta} \rightarrow[0,1]$ be a probability kernel such that $\mathbf{P}\left(x_{0}, \cdot\right)$ is the law of the space-time process associated with (1.1) on the time horizon $[0, \Delta]$ initialized from the point $\left(x_{0}, 0\right)$.

Note the following three properties of $x \mapsto \varphi_{j, k}(x)$ : i) Under assumption 1 , for all $k \geq 0, x \mapsto$ $g_{k}\left(x, y_{k}\right)$ is strictly positive and therefore so is $x \mapsto \varphi_{j, k}(x)$ for all $j \leq k$. ii) Under assumption 1 for all $k \geq 0, x \mapsto g_{k}\left(x, y_{k}\right)$ is a member of $C^{2}$, and combined with (2.3)-(2.4) and the fact that the solution of (1.1) satisfies $X_{(k+1) \Delta}=a+B X_{k \Delta}+\sigma \xi_{k+1}$ where $\xi_{k+1}=e^{\Delta \beta} \int_{k \Delta}^{(k+1) \Delta} e^{-(t-k \Delta) \beta} \mathrm{d} B_{t}$ is a Gaussian random variable and $a=e^{\Delta \beta} \int_{0}^{\Delta} e^{-t \beta} \alpha \mathrm{d} t, B=e^{\Delta \beta}$, this implies $x \mapsto \varphi_{j, k}(x)$ is a member of $C^{2}$ for all $j \leq k$. iii) By (2.6) in lemma 3 and the equivalence between conditions 1) and 2) in lemma 1 with $f$ there taken to be $\varphi_{j, k}$, we have $\log \varphi_{j, k}(x) \leq \log \varphi_{j, k}(0)+\nabla_{x} \log \varphi_{j, k}(0)^{T} x-\frac{\lambda_{g}\left(j, y_{j}\right)}{2}\|x\|^{2}$, hence $\varphi_{j, k}(x)$ grows no faster than $e^{c\|x\|}$ as $\|x\| \rightarrow \infty$ where $c=\left\|\nabla_{x} \log \varphi_{j, k}(0)\right\|$.

In the remainder of the proof of the lemma, $j, k$ are fixed as in the statement lemma, and the dependence of various quantities on $j, k$ and $y_{j}, \ldots, y_{k}$ is not shown in the notation. Appealing to the properties of $x \mapsto \varphi_{j, k}(x)$ which have just been stated, $x \mapsto h(x, t)$ is strictly positive, a member of $C^{2}$, and log-concave by lemma 3 and lemma 2 . With:

$$
D_{t}:=\frac{h\left(X_{t}, t\right)}{h\left(x_{0}, 0\right)}
$$


$\left(D_{t}\right)_{t \in[0, \Delta]}$ is a $\left(\mathcal{F}_{t}, \mathbf{P}\left(x_{0}, \cdot\right)\right)$-continuous martingale, and the expected value of $D_{t}$ under $\mathbf{P}\left(x_{0}, \cdot\right)$ is 1 . Now define the probability kernel $\mathbf{P}^{h}(x, \cdot):=D_{\Delta} \cdot \mathbf{P}(x, \cdot)$. Note that $\mathbf{P}^{h}$ depends on $j, k$ and $y_{j}, \ldots, y_{k}$ via $h$. Moreover under $\mathbf{P}^{h}\left(x_{0}, \cdot\right),\left(X_{t}\right)_{t \in[0, \Delta]}$ is an inhomogeneous Markov process with transition probabilities:

$$
P_{s, t}^{h}\left(x, \mathrm{~d} x^{\prime}\right):=\frac{P_{t-s}\left(x, \mathrm{~d} x^{\prime}\right) h\left(x^{\prime}, t\right)}{h(x, s)},
$$

and $R_{j, k}(x, A)=P_{0, \Delta}^{h}(x, A)=\mathbf{P}^{h}\left(x,\left\{X_{\Delta} \in A\right\}\right)$. By [23, Prop. 3.9, p.357], the extended generator of the space-time process under $\mathbf{P}^{h}\left(x_{0}, \cdot\right)$ is $h^{-1} L(h f)$. Using the fact that $\int P_{s}\left(x, \mathrm{~d} x^{\prime}\right) h\left(x^{\prime}, s+t\right)=h(x, t)$ we have $L(h)=0$, and combining this observation with elementary differential calculus manipulations it can be checked that $h^{-1} L(h f)$ is equal to the right hand side of (2.8).

Before stating the following proposition, we emphasize once again that $\left(y_{k}\right)_{k \in \mathbb{N}_{0}}$ are fixed.

Proposition 1. Fix any $j, k$ such that $1 \leq j \leq k$. If there exists a continuous function $\lambda_{h}:[0, \Delta] \rightarrow$ $[0, \infty)$ and a function $\tilde{h}: \mathbb{R}^{p} \times[0, \Delta] \rightarrow(0, \infty)$ such that for each $t, x \mapsto \tilde{h}(x, t)$ is log-concave and $h$ as in lemma 4 satisfies $h(x, t)=\exp \left[-\frac{\lambda_{h}(t)}{2}\|x\|^{2}\right] \tilde{h}(x, t)$, then for any $q \geq 1$,

$$
W_{q}\left(R_{j, k}(x, \cdot), R_{j, k}\left(x^{\prime}, \cdot\right)\right) \leq \exp \left[-\lambda_{\operatorname{sig}} \Delta-\sigma^{2} \int_{0}^{\Delta} \lambda_{h}(t) \mathrm{d} t\right]\left\|x-x^{\prime}\right\| .
$$

Proof. Consider the synchronous coupling:

$$
\begin{aligned}
& X_{t}=x_{0}+\int_{0}^{t} \alpha+\beta X_{s}+\sigma^{2} \nabla_{x} \log h\left(X_{s}, s\right) \mathrm{d} s+\sigma B_{t}, \\
& X_{t}^{\prime}=x_{0}^{\prime}+\int_{0}^{t} \alpha+\beta X_{s}^{\prime}+\sigma^{2} \nabla_{x} \log h\left(X_{s}^{\prime}, s\right) \mathrm{d} s+\sigma B_{t} .
\end{aligned}
$$

By Ito's formula, for any continuous function $\zeta:[0, \Delta] \rightarrow \mathbb{R}$.

$$
\begin{aligned}
& \left\|X_{t}-X_{t}^{\prime}\right\|^{2} e^{2 \int_{0}^{t} \zeta(s) \mathrm{d} s} \\
& \quad=\left\|x_{0}-x_{0}^{\prime}\right\|^{2}+2 \int_{0}^{t}\left(\zeta(s)\left\|X_{s}-X_{s}^{\prime}\right\|^{2}+\left(X_{s}-X_{s}^{\prime}\right)^{T} \beta\left(X_{s}-X_{s}^{\prime}\right)\right) e^{2 \int_{0}^{s} \zeta(u) \mathrm{d} u} \mathrm{~d} s \\
& \quad+2 \int_{0}^{t} \sigma^{2}\left(\nabla_{x} \log h\left(X_{s}, s\right)-\nabla_{x} \log h\left(X_{s}^{\prime}, s\right)\right)^{T}\left(X_{s}-X_{s}^{\prime}\right) e^{2 \int_{0}^{s} \zeta(u) \mathrm{d} u} \mathrm{~d} s .
\end{aligned}
$$

Now set $\zeta(s)=\lambda_{\text {sig }}+\sigma^{2} \lambda_{h}(s)$. For any skew-symmetric matrix, say $A$, and any $u \in \mathbb{R}^{p}, u^{T} A u=$ $(A u)^{T} u=u^{T} A^{T} u=-u^{T} A u$, hence $u^{T} A u=0$, so

$$
u^{T} \beta u=\frac{1}{2} u^{T}\left(\beta+\beta^{T}\right) u \leq-\lambda_{\text {sig }}\|u\|^{2}, \quad \forall u \in \mathbb{R}^{p} .
$$

The assumption of the lemma on $h$ combined with lemma 1 implies

$$
\left(\nabla_{x} \log h(x, s)-\nabla_{x} \log h\left(x^{\prime}, s\right)\right)^{T}\left(x-x^{\prime}\right) \leq-\lambda_{h}(s)\left\|x-x^{\prime}\right\|^{2}, \quad x, x^{\prime} \in \mathbb{R}^{p} .
$$

Applying (2.10) and (2.11) to (2.9) gives:

$$
\left\|X_{\Delta}-X_{\Delta}^{\prime}\right\| \leq \exp \left(-\int_{0}^{\Delta} \lambda_{\text {sig }}+\sigma^{2} \lambda_{h}(t) \mathrm{d} t\right)\left\|x_{0}-x_{0}^{\prime}\right\| .
$$

The proof is completed by taking expectations and applying lemma 4 . 


\subsection{Quantifying log-concavity of $x \mapsto h(x, t)$}

The main result of this section is proposition 2, which complements lemma 2 by quantifying the influence on the log-concavity of $x \mapsto h(x, t)$ of the parameters of the signal process and the logconcavity of the likelihood functions, and provides verification of the hypotheses of proposition 1.

Proposition 2. Let assumption 1 hold, fix $j, k$ such that $1 \leq j \leq k$ and let $h$ be as in lemma 4. Then there exists a function $\tilde{h}: \mathbb{R}^{p} \times[0, \Delta] \rightarrow(0, \infty)$ such that $x \mapsto \tilde{h}(x, t)$ is log-concave and

$$
h(x, t)=\exp \left[-\frac{\lambda_{h}(t)}{2}\|x\|^{2}\right] \tilde{h}(x, t)
$$

where

$$
\lambda_{h}(t):=\frac{\lambda_{g}\left(j, y_{j}\right) \lambda_{\beta}^{\min }(\Delta-t)}{1+\sigma^{2} \lambda_{g}\left(j, y_{j}\right) \int_{t}^{\Delta} \lambda_{\beta}^{\max }(\Delta-s) \mathrm{d} s},
$$

and $\lambda_{\beta}^{\min }(t), \lambda_{\beta}^{\max }(t)$ are respectively the minimum and maximum eigenvalues of $e^{\beta t}\left(e^{\beta t}\right)^{T}$.

We shall make use of the following well-known lemma [21, Thm. 6].

Lemma 5. For every function $(u, v) \mapsto f(u, v)$ on $\mathbb{R}^{p} \times \mathbb{R}^{q}$ which is log-concave in $(u, v)$, the integral $\int f(u, v) \mathrm{d} v$ is a log-concave function of $u$.

Lemma 6 and lemma 7 are technical results used in the proof of proposition 2.

Lemma 6. Let $F, S$ be real, square, symmetric matrices such that $F+S$ is invertible. Then

$$
v^{T} F v+(u-v)^{T} S(u-v)=u^{T} C u+z^{T}(F+S) z
$$

where $C:=F(F+S)^{-1} S$ and $z:=v-(F+S)^{-1} S u$.

Proof. We have using the assumed symmetry of $F$ and $S$,

$$
z^{T}(F+S) z=v^{T}(F+S) v-2 u^{T} S v+u^{T} S(F+S)^{-1} S u .
$$

Therefore

$$
\begin{aligned}
u^{T} C u+z^{T}(F+S) z & =u^{T} S u+v^{T}(F+S) v-2 u^{T} S v \\
& =v^{T} F v+(u-v)^{T} S(u-v) .
\end{aligned}
$$

Lemma 7. Let $f$ be any function of the form $f(u): u \in \mathbb{R}^{p} \mapsto \exp \left(-\frac{1}{2} u^{T} F u\right) \tilde{f}(u)$ where $F$ is a real symmetric matrix and $\tilde{f}$ is log-concave, and let $S$ be a real symmetric matrix such that $F+S$ is invertible. Then for any $a \in \mathbb{R}^{p}$ and $p \times p$ real matrix $B$,

$$
f(v) \exp \left[-\frac{1}{2}(v-a-B u)^{T} S(v-a-B u)\right]=\exp \left(-\frac{1}{2} u^{T} B^{T} C B u\right) \tilde{f}(v) \exp \left[-\frac{1}{2} z^{T}(F+S) z\right],
$$

where $C=F(F+S)^{-1} S$ and $z=v-(F+S)^{-1} S(a+B u)$

Proof. Using lemma 6 with $u$ there replaced by $a+B u$,

$$
\begin{aligned}
& f(v) \exp \left[-\frac{1}{2}(v-a-B u)^{T} S(v-a-B u)\right] \\
& =\tilde{f}(v) \exp \left[-\frac{1}{2}\left\{v^{T} F v+(v-a-B u)^{T} S(v-a-B u)\right\}\right] \\
& =\tilde{f}(v) \exp \left[-\frac{1}{2}\left\{(a+B u)^{T} C(a+B u)+z^{T}(F+S) z\right\}\right] \\
& =\exp \left(-\frac{1}{2} u^{T} B^{T} C B u\right) \exp \left[-\frac{1}{2}\left(a^{T} C a+2 a^{T} C B u\right)\right] \tilde{f}(v) \exp \left[-\frac{1}{2} z^{T}(F+S) z\right] .
\end{aligned}
$$


Proof of proposition 2. First note that for the signal process $\left(X_{t}\right)_{t \in \mathbb{R}_{+}}$as per (1.1),

$$
\begin{aligned}
& m_{t}:=\mathbf{E}_{x_{0}}\left[X_{t}\right]=a_{t}+e^{\beta t} x_{0} \\
& \Sigma_{t}:=\mathbf{E}_{x_{0}}\left[\left(X_{t}-m_{t}\right)\left(X_{t}-m_{t}\right)^{T}\right]=\sigma^{2} \int_{0}^{t} e^{\beta(t-s)}\left(e^{\beta(t-s)}\right)^{T} \mathrm{~d} s
\end{aligned}
$$

where

$$
a_{t}:=e^{\beta t} \int_{0}^{t}\left(e^{\beta s}\right)^{-1} \alpha \mathrm{d} s
$$

It follows that $u^{T} \Sigma_{t}^{-1} u \geq \Lambda_{t}^{-1} u^{T} u$ for all $u \in \mathbb{R}^{p}$ with the shorthand $\Lambda_{t}:=\sigma^{2} \int_{0}^{t} \lambda_{\beta}^{\max }(s) \mathrm{d} s$.

Applying lemma 7 with $a=a_{t}, B=e^{\beta t}, S=I \Lambda_{t}^{-1}, f=\varphi_{j, k}, F=I \lambda_{g}\left(j, y_{j}\right)$, and lemma 3 ,

$$
\begin{aligned}
& \varphi_{j, k}(x) \exp \left[-\frac{1}{2}\left(x-a_{t}-e^{\beta t} x_{0}\right)^{T} \Sigma_{t}^{-1}\left(x-a_{t}-e^{\beta t} x_{0}\right)\right] \\
& =\exp \left(-\frac{1}{2} \frac{\lambda_{g}\left(j, y_{j}\right) \lambda_{\beta}^{\min }(t)}{1+\lambda_{g}\left(j, y_{j}\right) \Lambda_{t}} x_{0}^{T} x_{0}\right) \\
& \cdot \tilde{\varphi}_{j, k}(x) \exp \left[-\frac{1}{2} z_{t}^{T} z_{t}\left(\lambda_{g}\left(j, y_{j}\right)+\Lambda_{t}^{-1}\right)\right] \\
& \cdot \exp \left[-\frac{1}{2} \frac{\lambda_{g}\left(j, y_{j}\right)}{1+\lambda_{g}\left(j, y_{j}\right) \Lambda_{t}} x_{0}^{T}\left(\left(e^{\beta t}\right)^{T} e^{\beta t}-I \lambda_{\beta}^{\min }(t)\right) x_{0}\right] \\
& \cdot \exp \left[-\frac{1}{2}\left(x-a_{t}-e^{\beta t} x_{0}\right)^{T}\left(\Sigma_{t}^{-1}-\Lambda_{t}^{-1} I\right)\left(x-a_{t}-e^{\beta t} x_{0}\right)\right],
\end{aligned}
$$

where $z_{t}=x-\left(a_{t}+e^{\beta t} x_{0}\right) /\left(1+\lambda_{g}\left(j, y_{j}\right) \Lambda_{t}\right)$.

The product of the terms in (2.12)-(2.14) is jointly log-concave in $\left(x_{0}, x\right)$. Therefore by lemma 5 , there exists a function $\tilde{h}$ such that $x \mapsto \tilde{h}(x, t)$ is log-concave and

$$
\begin{aligned}
h\left(x_{0}, t\right) & =P^{\Delta-t} \varphi_{j, k}\left(x_{0}\right) \\
& =\int \varphi_{j, k}(x) \exp \left[-\frac{1}{2}\left(x-a_{\Delta-t}-e^{\beta(\Delta-t)} x_{0}\right)^{T} \Sigma_{\Delta-t}^{-1}\left(x-a_{\Delta-t}-e^{\beta(\Delta-t)} x_{0}\right)\right] \mathrm{d} x \\
& =\exp \left(-\frac{1}{2} \frac{\lambda_{g}\left(j, y_{j}\right) \lambda_{\beta}^{\min }(\Delta-t)}{1+\lambda_{g}\left(j, y_{j}\right) \Lambda_{\Delta-t}} x_{0}^{T} x_{0}\right) \tilde{h}\left(x_{0}, t\right),
\end{aligned}
$$

which completes the proof.

\subsection{Discussion of theorem 1}

The aim of this section is to help interpret the quantities on the right hand side of (2.1) and their combined effect on the behaviour of (2.1) as $k$ grows.

\subsubsection{Dimension-free nature of the contraction rate}

The quantity $\lambda(j, y, t)$ in $(2.2)$ does not necessarily depend on the dimension of the state space, $\mathbb{R}^{p}$. For example, the quantities $\lambda_{g}(j, y), \lambda_{\mathrm{sig}}, \lambda_{\beta}^{\min }(t), \lambda_{\beta}^{\max }(t)$ and $\sigma^{2}$ appearing in (2.2) are stable under tensor products of the model described in section 1 , in the sense that if one expands the model to state-space $\mathbb{R}^{2 p}$ by defining the signal to be two independent and identically distributed copies of (1.1), independently observed as $y_{k}=\left[y_{k}^{(1)} y_{k}^{(2)}\right] \in \mathbb{Y}^{2}$ with likelihood functions having common strong $\log$-concavity parameter $\lambda_{g}\left(k, y_{k}\right)$, then there is no degradation of $\lambda(j, y, t)$. To make this precise note that: 
1) $\begin{aligned} g_{k}\left(x^{(i)}, y_{k}^{(i)}\right)=\exp \left[-\frac{\lambda_{g}\left(k, y_{k}\right)}{2}\left\|x^{(i)}\right\|^{2}\right] \tilde{g}_{k}\left(x^{(i)}, y_{k}^{(i)}\right), \quad i=1,2, \\ \Longrightarrow \quad g_{k}\left(x^{(1)}, y_{k}^{(1)}\right) g_{k}\left(x^{(2)}, y_{k}^{(2)}\right)=\exp \left[-\frac{\lambda_{g}\left(k, y_{k}\right)}{2}\left(\left\|x^{(1)}\right\|^{2}+\left\|x^{(2)}\right\|^{2}\right)\right] \tilde{g}_{k}\left(x^{(1)}, y_{k}^{(1)}\right) \tilde{g}_{k}\left(x^{(2)}, y_{k}^{(2)}\right),\end{aligned}$

2) $\operatorname{spectrum}\left\{\left(\beta+\beta^{T}\right) / 2\right\}=\operatorname{spectrum}\left\{\left(\beta^{\otimes 2}+\left(\beta^{\otimes 2}\right)^{T}\right) / 2\right\}$,

3) $\operatorname{spectrum}\left\{e^{\beta t}\left(e^{\beta t}\right)^{T}\right\}=\operatorname{spectrum}\left\{e^{\beta^{\otimes 2} t}\left(e^{\beta^{\otimes 2} t}\right)^{T}\right\}$,

where $\beta^{\otimes 2}$ denotes the Kronecker product $\left[\begin{array}{ll}1 & 0 \\ 0 & 1\end{array}\right] \otimes \beta$. 1) shows that $x=\left[x^{(1)} x^{(2)}\right]^{T} \mapsto g_{k}\left(x^{(1)}, y_{k}^{(1)}\right) g_{k}\left(x^{(2)}, y_{k}^{(2)}\right)$ is strongly log-concave with parameter $\lambda_{g}\left(k, y_{k}\right)$. 2) and 3) imply that $\lambda_{\text {sig }}, \lambda_{\beta}^{\min }(t), \lambda_{\beta}^{\max }(t)$ are preserved by expanding the model from $\mathbb{R}^{p}$ to $\mathbb{R}^{2 p}$ in the above stated fashion.

\subsubsection{The relationship between signal stability and filter stability}

For the signal model (1.1) in general, $\lambda_{\text {sig }}$ could be negative, zero or positive. When $\lambda_{\text {sig }}>0$ the signal is exponentially stable, as follows from:

Lemma 8. For any given $\alpha, \beta, \sigma$, the transition probabilities $P_{t}(x, \cdot):=\mathbf{P}\left(X_{t} \in \cdot \mid X_{0}=x\right)$ of the signal model (1.1) satisfy, for any $q \geq 1$,

$$
W_{q}\left(P_{t}(x, \cdot), P_{t}\left(x^{\prime}, \cdot\right)\right) \leq \exp \left(-\lambda_{\text {sig }} t\right)\left\|x-x^{\prime}\right\|, \quad \forall x, x^{\prime} \in \mathbb{R}^{p} .
$$

Proof of lemma 8. The proof follows the same synchronous coupling argument used in the proof of proposition 1 but with the $\nabla_{x} \log h$ term there replaced by zero, so the details are omitted.

The inequality (2.15) cannot be improved in general. For example, in the case that $p=1$, we have $\beta=-\lambda_{\text {sig }}, P_{t}(x, \cdot)=\mathcal{N}\left(m_{t}(x), \nu_{t}\right)$ where $\dot{m}_{t}(x)=\alpha+\beta m_{t}(x)$ with $m_{0}(x)=x, \dot{\nu}_{t}=2 \beta \nu_{t}+\sigma^{2}$ with $\nu_{0}=0$, and the order $q=2$ Wasserstein distance is available in closed form [10, eq. 3]:

$$
W_{2}\left(P_{t}(x, \cdot), P_{t}\left(x^{\prime}, \cdot\right)\right)=\left|m_{t}(x)-m_{t}\left(x^{\prime}\right)\right|=\exp \left(-\lambda_{\text {sig }} t\right)\left|x-x^{\prime}\right| .
$$

Thus when $\lambda_{\text {sig }} \leq 0$ the signal is not exponentially stable in general.

Now let us turn to the question of how $\lambda_{\text {sig }}$ impacts filter stability. Inspecting (2.2) we observe that the ratio on the right hand side is always nonnegative, because $\lambda_{g}(k, y) \geq 0$ for all $k$ and $y$ under assumption 1 , and $e^{\beta t}\left(e^{\beta t}\right)^{T}$ is symmetric and positive semidefinite. Therefore with no further assumptions than those of theorem 1 the following bound holds for any $q \geq 1$,

$$
W_{q}\left(\pi_{k}\left(x, y_{0: k}, \cdot\right), \pi_{k}\left(x^{\prime}, y_{0: k}, \cdot\right)\right) \leq \exp \left(-\lambda_{\text {sig }} k \Delta\right)\left\|x-x^{\prime}\right\|, \quad \forall x, x^{\prime} \in \mathbb{R}^{p}, y_{0}, \ldots, y_{k} \in \mathbb{Y} .
$$

Thus when $\lambda_{\text {sig }}>0$, the filter inherits exponential stability from the signal. The ratio term in (2.2) determines whether or not we can deduce a tighter bound than (2.17) from (2.1), and in particular determines whether or not the right hand side of (2.1) converges to zero as $k \rightarrow \infty$ when $\lambda_{\text {sig }} \leq 0$.

Introducing a simplifying assumption that $\beta$ is a diagonal matrix allows us to derive a more easily interpretable upper bound for this ratio term, which we shall examine in the context of specific observation models below.

Lemma 9. In addition to assumption 1 , let $\beta$ be a diagonal matrix with maximum and minimum diagonal elements respectively denoted $\bar{\beta}, \underline{\beta} \in \mathbb{R}$. Then for any $q \geq 1, k \geq 1$ and $y_{0}, \ldots, y_{k} \in \mathbb{Y}$,

$$
\begin{aligned}
& W_{q}\left(\pi_{k}\left(x, y_{0: k}, \cdot\right), \pi_{k}\left(x^{\prime}, y_{0: k}, \cdot\right)\right) \leq \\
& \exp \left[k \Delta \bar{\beta}-\sum_{j=0}^{k} e^{-2 \Delta(\bar{\beta}-\underline{\beta})} \log \left[1+\frac{\sigma^{2} \lambda_{g}\left(j, y_{j}\right)}{2 \bar{\beta}}\left(e^{2 \bar{\beta} \Delta}-1\right)\right]\right]\left\|x-x^{\prime}\right\|, \quad \forall x, x^{\prime} \in \mathbb{R}^{p} .
\end{aligned}
$$


Proof. Starting from the identity

$$
\frac{\sigma^{2} \lambda_{g}(j, y) \lambda_{\beta}^{\min }(\Delta-t)}{1+\sigma^{2} \lambda_{g}(j, y) \int_{t}^{\Delta} \lambda_{\beta}^{\max }(\Delta-s) \mathrm{d} s}=\frac{\lambda_{\beta}^{\min }(\Delta-t)}{\lambda_{\beta}^{\max }(\Delta-t)}\left(-\frac{\mathrm{d}}{\mathrm{d} t} \log \left[1+\sigma^{2} \lambda_{g}(j, y) \int_{t}^{\Delta} \lambda_{\beta}^{\max }(\Delta-s) \mathrm{d} s\right]\right),
$$

then integrating by parts and using the fact that under the diagonal assumption on $\beta, \lambda_{\beta}^{\min }(t)=$ $e^{2 t \underline{\beta}}, \lambda_{\beta}^{\max }(t)=e^{2 t \bar{\beta}}$

$$
\begin{aligned}
& \int_{0}^{\Delta} \frac{\sigma^{2} \lambda_{g}(j, y) \lambda_{\beta}^{\min }(\Delta-t)}{1+\sigma^{2} \lambda_{g}(j, y) \int_{t}^{\Delta} \lambda_{\beta}^{\max }(\Delta-s) \mathrm{d} s} \mathrm{~d} t \\
& =\frac{\lambda_{\beta}^{\min }(\Delta)}{\lambda_{\beta}^{\max }(\Delta)} \log \left[1+\sigma^{2} \lambda_{g}(j, y) \int_{0}^{\Delta} e^{2(\Delta-s) \bar{\beta}} \mathrm{d} s\right] \\
& +2(\bar{\beta}-\underline{\beta}) \int_{0}^{\Delta} e^{-2(\Delta-t)(\bar{\beta}-\underline{\beta})} \log \left[1+\sigma^{2} \lambda_{g}(j, y) \int_{t}^{\Delta} e^{2(\Delta-s) \bar{\beta}} \mathrm{d} s\right] \mathrm{d} t \\
& \geq e^{-2 \Delta(\bar{\beta}-\underline{\beta})} \log \left[1+\frac{\sigma^{2} \lambda_{g}(j, y)}{2 \bar{\beta}}\left(e^{2 \bar{\beta} \Delta}-1\right)\right],
\end{aligned}
$$

where the lower bound holds by computing the integral on the right hand side of (2.18) and using the fact that (2.19) is nonnegative. The proof is completed by substituting the lower bound (2.20) into the result of theorem 1 and noting that under the diagonal assumption on $\beta, \lambda_{\text {sig }}=-\bar{\beta}$.

\subsubsection{Examples}

\section{Linear-Gaussian observations}

In this case $\mathbb{Y}=\mathbb{R}^{n}$ and for all $k \in \mathbb{N}_{0}$,

$$
g_{k}(x, y)=(2 \pi)^{-n / 2} \operatorname{det}(\Sigma)^{-1 / 2} \exp \left[-\frac{1}{2}(y-A x)^{T} \Sigma^{-1}(y-A x)\right],
$$

where $A$ and $\Sigma$ are matrices of appropriate sizes and $\Sigma$ is symmetric and positive definite. For any $u \in \mathbb{R}^{p}, u^{T} A^{T} \Sigma^{-1} A u \geq\|u\|^{2} \lambda_{A^{T} A}^{\min } / \lambda_{\Sigma}^{\max }$ where $\lambda_{A^{T} A}^{\min }$ is the minimum eigenvalue of $A^{T} A$ and $\lambda_{\Sigma}^{\max }$ is the maximum eigenvalue of $\Sigma$. Thus for $(2.21)$, assumption 1 holds with $\lambda_{g}(k, y)$ taken to be $\lambda_{A^{T} A}^{\min } / \lambda_{\Sigma}^{\max }$ for all $k$ and $y$.

For ease of exposition, consider the case of diagonal $\beta$ addressed in lemma 9. If $\lambda_{A^{T} A}^{\min }=0$, i.e. $A$ is rank-deficient, and taking $\lambda_{g}(k, y)=\lambda_{A^{T} A}^{\min } / \lambda_{\Sigma}^{\max }$, the right hand side of the bound in lemma 9 tends to zero as $k \rightarrow \infty$ only if $\lambda_{\text {sig }}=-\bar{\beta}>0$, i.e. if the signal is stable. On the other hand if again one takes $\lambda_{g}(k, y)=\lambda_{A^{T} A}^{\min } / \lambda_{\Sigma}^{\max }$, but now with some fixed $\lambda_{A^{T} A}^{\min }>0$ and $\lambda_{\text {sig }}=-\bar{\beta} \leq 0$, the right hand side of the bound of lemma 9 tends to zero as $k \rightarrow \infty$ if $\sigma^{2} / \lambda_{\Sigma}^{\max }$ is large enough, which means that the level of noise in the observations is small relative to the level of noise in the signal.

As an example of how the filter can indeed fail to be stable if $\lambda_{A^{T} A}^{\min } \leq 0$, consider the case in which $p=2, \beta=\left[\begin{array}{cc}\beta^{(1)} & 0 \\ 0 & \beta^{(2)}\end{array}\right]$ for any $\beta^{(1)}, \beta^{(2)} \in \mathbb{R}, n=1$ and $A=[01]$, so the first coordinate of the signal is completely unobserved. In this scenario it follows from (1.2) that $\pi_{k}\left(x, y_{0: k}, A \times \mathbb{R}\right)=$ $\tilde{P}_{k \Delta}\left(x^{(1)}, A\right)$, for any $A \in \mathcal{B}(\mathbb{R}), x=\left[x^{(1)} x^{(2)}\right]^{T} \in \mathbb{R}^{2}$ and where $\left(\tilde{P}_{t}\right)_{t \geq 0}$ are the transition probabilities of the first coordinate of the signal process, i.e. the solution of $\mathrm{d} X_{t}^{(1)}=\left(\alpha^{(1)}+\beta^{(1)} X_{t}^{(1)}\right) \mathrm{d} t+\sigma \mathrm{d} B_{t}^{(1)}$. Therefore, using (2.16), the filter is not exponentially stable if $\beta^{(1)} \geq 0$.

The pair of conditions that either i) $\lambda_{\text {sig }}>0$, or that ii) $\lambda_{A^{T} A}^{\min }>0$ and $\sigma^{2} / \lambda_{\Sigma}^{\max }$ is large enough, are together qualitatively similar to the notion of "detectability" in linear systems theory and in terms of which stability of the Kalman filter can be established, see e.g. [29] for a summary and historical 
references. However it does not seem easy to make a close comparison to the stability results surveyed in [29] because they concern the total variation distance and involve the observations being random and generated by the model. By contrast heorem 1 concerns the Wasserstein distance and subject to assumption 1, the obserations are arbitrary.

\section{Stochastic volatility}

In this case $\mathbb{Y}=\mathbb{R}^{p}$, and for all $k \in \mathbb{N}_{0}$,

$$
g_{k}(x, y)=(2 \pi)^{-p / 2} \operatorname{det}(V(x))^{1 / 2} \exp \left[-\frac{1}{2} y^{T} V(x) y\right], \quad V(x)=\operatorname{diag}\left\{\exp \left(-x^{(1)}\right), \cdots, \exp \left(-x^{(p)}\right)\right\},
$$

where $x=\left[x^{(1)} \cdots x^{(p)}\right]^{T} \in \mathbb{R}^{p}$. Stochastic volatility models are very popular in econometrics and finance $[14,20,13]$. The observations $\left(y_{k}\right)_{k \in \mathbb{N}_{0}}$, where $y_{k}=\left[y_{k}^{(1)} \cdots y_{k}^{(p)}\right]^{T}$, represent the returns on a family of $p$ financial assets, whose time varying volatilities are modelled through the signal process. Writing out the log-likelihood function:

$$
\log g_{k}(x, y)=-\frac{p}{2} \log 2 \pi-\frac{1}{2} \sum_{i=1}^{p} x^{(i)}-\frac{1}{2} \sum_{i=1}^{p}\left(y^{(i)}\right)^{2} \exp \left(-x^{(i)}\right),
$$

it is readily checked that assumption 1 is satisfied with $\lambda_{g}(k, y)=0$, for all $k \in \mathbb{N}_{0}$ and $y \in \mathbb{Y}$. Therefore for this stochastic volatility model we need to rely on the condition $\lambda_{\text {sig }}>0$ in order to deduce that the right hand side of (2.1) tends to zero as $k \rightarrow \infty$. However it is remarkable that such convergence holds without any on the realized observations $y_{0}, \ldots, y_{k}$, compared to analogous results for stochastic volatility models which concern total variation rather than Wasserstein distance, e.g [7, Sec 4.3], in which certain stochastic hypotheses are placed on the observation sequence in order to prove that the filter forgets its initial condition almost surely with respect to the law of the observations.

\section{Markov random field model for neural data}

In statistical neuroscience, log-concave likelihood functions appear in Markov random field models used to analyze time-varying correlations in multivariate neural spike trains [24,6]. Here $y_{k} \in \mathbb{Y}=\{0,1\}^{n}$ is a binary vector indicating the firing pattern $n$ neurons in the $k$ th time window, and

$$
g_{k}(x, y)=\exp \left\{\sum_{i=1}^{n} \sum_{j>i} y^{(i)} y^{(j)} x^{(i, j)}+\sum_{i=1}^{n} y^{(i)} x^{(i)}-\psi(x)\right\},
$$

where $p=n(n-1)+n, x$ is a vector with elements $\left\{x^{(i, j)} ; j>i, x^{(i)} ; i=1, \ldots, n\right\}$, and $\psi$, called the log-partition function, is smooth and convex. Assumption 1 holds with $\lambda_{g}(k, y)=0$ for all $k \in \mathbb{N}_{0}$ and $y \in \mathbb{Y}$.

\section{Exponential families and dynamic generalized linear models}

The observation models in (2.21) and (2.22), as distributions over $y$ parameterized by $x$, are socalled exponential families of distributions [26]. Other exponential families include the beta, Dirichlet, exponential, Fisher, gamma, Multinomial, Poisson and Von Mises distributions, to mention just a few. It is a property of exponential families that their log-likelihood function, as a function of their canonical parameter, is smooth and log-concave [26, Prop 3.10]. Thus whenever $g_{k}(x, y)$ is an exponential family of distributions over $y$ with canonical parameter $x \in \mathbb{R}^{p}$, and $x \mapsto g_{k}(x, y)$ is strictly positive for all $k \in \mathbb{N}_{0}, y \in \mathbb{Y}$, assumption 1 holds with $\lambda_{g}(k, y)=0$ for all $k \in \mathbb{N}_{0}$ and $y \in \mathbb{Y}$.

Exponential families of distributions form the building blocks of Generalized Linear Models [17]. In this setting $y_{k}=\left[y_{k}^{(1)} \cdots y_{k}^{(n)}\right]^{T} \in \mathbb{Y} \subseteq \mathbb{R}^{n}$ is a vector of response variables whose relationship with 
covariates $z_{k}=\left(z_{k}^{(i, j)}\right), i=1, \ldots, n, j=1, \ldots, p$, is modelled through $g_{k}\left(x, y_{k}\right)$ of the form:

$$
g_{k}\left(x, y_{k}\right)=\exp \left[\sum_{i=1}^{n}\left\{\sum_{j=1}^{p} y_{k}^{(i)} z_{k}^{(i, j)} x^{(j)}-\psi\left(\sum_{j=1}^{p} z_{k}^{(i, j)} x^{(j)}\right)+\log \phi\left(y_{k}^{(i)}\right)\right\}\right],
$$

where $x=\left[x^{(1)} \cdots x^{(p)}\right]^{T}$ is the vector of regression parameters, $\phi$ is a given function, and $\psi$ is convex, so that $x \mapsto g_{k}\left(x, y_{k}\right)$ is indeed log-concave. The situation in which the regression parameter $x$ is treated as time-varying is known as a Dynamic Generalized Linear Model [12]. An example is discussed in section 3 .

\section{Smoothing distributions and a family of weighted Wasserstein distances}

It appears to be a nontrivial matter to extend theorem 1 to the case where the filter is initialized from two general probability measures, say $\mu$ and $\nu$ on $\mathcal{B}\left(\mathbb{R}^{p}\right)$ instead of only at points $x$ and $x^{\prime}$, in a way which can yield a contractive bound in terms of $W_{q}(\mu, \nu)$. The difficulty stems from the fact that the generalization of (2.5) to an arbitrary initial distribution $\mu$ is, with a slight overloading of the notation $\pi_{k}$ in its first argument:

$$
\pi_{k}\left(\mu, y_{0: k}, A\right):=\mu_{0, k} R_{1, k} R_{2, k} \cdots R_{k, k}(A), \quad \mu_{0, k}(A):=\frac{\mu \cdot \varphi_{0, k}}{\mu \varphi_{0, k}},
$$

where the dependence of $\mu_{0, k}$ on $y_{0: k}$ is not shown in the notation. A direct corollary of theorem 1 together with the identity (2.5) is:

$$
W_{q}\left(\pi_{k}\left(\mu, y_{0: k}, \cdot\right), \pi_{k}\left(\nu, y_{0: k}, \cdot\right)\right) \leq \exp \left[-\sum_{j=1}^{k} \int_{0}^{\Delta} \lambda\left(j, y_{j}, t\right) \mathrm{d} t\right] W_{q}\left(\mu_{0, k}, \nu_{0, k}\right),
$$

but even if $\lim _{k \rightarrow \infty} \exp \left[-\sum_{j=1}^{k} \int_{0}^{\Delta} \lambda\left(j, y_{j}, t\right) \mathrm{d} t\right]=0$, it cannot be deduced immediately from (3.2) that the left hand side of (3.2) converges to zero as $k \rightarrow \infty$ due to the dependence of $W_{q}\left(\mu_{0, k}, \nu_{0, k}\right)$ on $k$ and $y_{0: k}$.

An alternative is to work with a certain family of weighted Wasserstein distances between filtering distributions. As we shall see, this is equivalent to establishing forgetting of the initial condition for so-called smoothing distributions, which unlike filtering distributions condition on future as well as past and present observations. To describe this equivalence in more detail we shall need the following lemma.

Lemma 10. Let $d(\cdot, \cdot)$ be a metric on the set of probability measures on $\mathcal{B}\left(\mathbb{R}^{p}\right)$ and let $\phi: \mathbb{R}^{p} \rightarrow(0, \infty)$. Then $d^{\phi}(\cdot, \cdot)$ defined by:

$$
d^{\phi}:(\mu, \nu) \longmapsto d\left(\frac{\mu \cdot \phi}{\mu \phi}, \frac{\nu \cdot \phi}{\nu \phi}\right)
$$

is a metric on the subset of probability measures $\left\{\mu\right.$ on $\left.\mathcal{B}\left(\mathbb{R}^{p}\right): \mu \phi<\infty\right\}$.

Proof. It follows immediately from the assumption that $d$ is a metric and $\phi$ is strictly positive that on the given domain $\{\mu: \mu \phi<\infty\}, d^{\phi}$ is nonnegative, symmetric, satisfies the triangle inequality and $\mu=\nu \Rightarrow d^{\phi}(\mu, \nu)=0$. For the reverse implication, using the implication $d^{\phi}(\mu, \nu)=0 \Rightarrow \mu^{\phi}:=\frac{\mu \cdot \phi}{\mu \phi}=$ $\frac{\nu \cdot \phi}{\nu \phi}=: \nu^{\phi}$ and the strict positivity of $\phi$, we have $1=\mathrm{d} \mu^{\phi} / \mathrm{d} \nu^{\phi}=(\mathrm{d} \mu / \mathrm{d} \nu)(\nu \phi / \mu \phi), \nu$-a.e. Thus $\mathrm{d} \mu / \mathrm{d} \nu$ is a constant $\nu$-a.e. and since $\mu$ and $\nu$ are probability measures, it follows that if $d^{\phi}(\mu, \nu)=0$ then $\mu=\nu$. 
Throughout the remainder of section $3\left(y_{k}\right)_{k \in \mathbb{N}_{0}}$ are an arbitrarily chosen and then fixed sequence of observations, unless stated otherwise. To avoid cumbersome formulae, the dependence of some quantities on $\left(y_{k}\right)_{k \in \mathbb{N}_{0}}$ is not shown in the notation.

Let us introduce the nonnegative integral kernels

$$
Q_{k}\left(x, \mathrm{~d} x^{\prime}\right):=g_{k-1}\left(x, y_{k-1}\right) P_{\Delta}\left(x, \mathrm{~d} x^{\prime}\right) . \quad k \geq 1 . \quad Q_{j, k}:=Q_{j+1} \cdots Q_{k}, \quad 0 \leq j<k .
$$

and the probability measures

$$
\eta_{k}^{\mu}(A):=\frac{\mu Q_{0, k}\left(\mathbf{1}_{A}\right)}{\mu Q_{0, k}\left(\mathbf{1}_{\mathbb{R}^{p}}\right)}, \quad k \geq 1, \quad \eta_{0}^{\mu}:=\mu, \quad A \in \mathcal{B}\left(\mathbb{R}^{p}\right),
$$

for any $\mu$ such that the denominator is finite. We shall use the shorthand

$$
\pi_{k}^{\mu}(\cdot):=\pi_{k}\left(\mu, y_{0: k}, \cdot\right)
$$

The dependence of $Q_{k}$ on $y_{k-1}$, of $Q_{j, k}$ on $y_{j}, \ldots, y_{k-1}$, of $\eta_{k}^{\mu}$ on $y_{0}, \ldots, y_{k-1}$ and of $\pi_{k}^{\mu}$ on $y_{0}, \ldots, y_{k}$ is not shown in the notation. Note from (3.1) that $\eta_{k}^{\mu}(\cdot)=\pi_{k-1}^{\mu} P_{\Delta}(\cdot)$.

We shall use the functions appearing in the following assumption to define a family of weighted Wasserstein distances.

Assumption 2. There exists a probability measure $\mu_{0}$ such that for the given sequence $\left(y_{k}\right)_{k \in \mathbb{N}_{0}}$, the following pointwise limit exists for each $k \in \mathbb{N}_{0}$ :

$$
\phi_{k, \infty}(x):=\lim _{\ell \rightarrow \infty} \frac{\varphi_{k, \ell}(x)}{\eta_{k}^{\mu_{0}} \varphi_{k, \ell}}
$$

$\phi_{k, \infty}(x) \in(0, \infty)$ for all $x \in \mathbb{R}^{p}$, and the functions $\left(\phi_{k, \infty}\right)_{k \in \mathbb{N}_{0}}$ so-defined belong to $C^{2}$ and satisfy

$$
Q_{k} \phi_{k, \infty}=\varsigma_{k-1} \phi_{k-1, \infty}, \quad k \geq 1,
$$

where $\varsigma_{k}:=\int \eta_{k}^{\mu_{0}}(\mathrm{~d} x) g_{k}\left(x, y_{k}\right) \in(0, \infty)$.

Before discussing the interpretation of assumption 2, consider the following lemma, which mirrors lemma 3 .

Lemma 11. If assumption 2 holds, then for any $\mu$ such that for all $k \in \mathbb{N}_{0}, \pi_{k}^{\mu} P_{\Delta} \phi_{k+1, \infty}<\infty$, the probability measures $\left(\pi_{k, \infty}^{\mu}\right)_{k \in \mathbb{N}_{0}}$ defined by:

$$
\pi_{k, \infty}^{\mu}(A):=\frac{\pi_{k}^{\mu}\left(\mathbf{1}_{A} P_{\Delta} \phi_{k+1, \infty}\right)}{\pi_{k}^{\mu} P_{\Delta} \phi_{k+1, \infty}}, \quad A \in \mathcal{B}\left(\mathbb{R}^{p}\right)
$$

satisfy

$$
\pi_{k, \infty}^{\mu}(A)=\pi_{0, \infty}^{\mu} R_{1, \infty} \cdots R_{k, \infty}(A)
$$

with the Markov kernels

$$
R_{k, \infty}\left(x, \mathrm{~d} x^{\prime}\right):=\frac{P_{\Delta}\left(x, \mathrm{~d} x^{\prime}\right) \phi_{k, \infty}\left(x^{\prime}\right)}{P_{\Delta} \phi_{k, \infty}(x)} .
$$

If additionally assumption 1 holds, then for each $k \in \mathbb{N}_{0}$, there exists a log-concave function $\tilde{\phi}_{k, \infty}$ such that

$$
\phi_{k, \infty}(x)=\exp \left[-\frac{\lambda_{g}\left(k, y_{k}\right)}{2}\|x\|^{2}\right] \tilde{\phi}_{k, \infty}(x)
$$


Proof. To establish (3.7) it suffices to show $\pi_{k-1, \infty}^{\mu} R_{k, \infty}=\pi_{k, \infty}^{\mu}$. We have

$$
\begin{aligned}
\pi_{k-1, \infty}^{\mu} R_{k, \infty}(A) & =\frac{\pi_{k-1}^{\mu}\left(P_{\Delta}\left(\phi_{k, \infty}\right) R_{k, \infty}\left(\mathbf{1}_{A}\right)\right)}{\pi_{k-1}^{\mu} P_{\Delta k} \phi_{k, \infty}} \\
& =\frac{\pi_{k-1}^{\mu} P_{\Delta}\left(\mathbf{1}_{A} \phi_{k, \infty}\right)}{\pi_{k-1}^{\mu} P_{\Delta} \phi_{k, \infty}} \\
& =\frac{\pi_{k-1}^{\mu} P_{\Delta}\left(\mathbf{1}_{A} Q_{k+1} \phi_{k+1, \infty}\right)}{\pi_{k-1}^{\mu} P_{\Delta} Q_{k+1} \phi_{k+1, \infty}} \\
& =\frac{\pi_{k}^{\mu}\left(\mathbf{1}_{A} P_{\Delta} \phi_{k+1, \infty}\right)}{\pi_{k}^{\mu} P_{\Delta} \phi_{k+1, \infty}}=\pi_{k, \infty}^{\mu}(A),
\end{aligned}
$$

where (3.5), (3.3) and the identity $\pi_{k}^{\mu}(A)=\pi_{k-1}^{\mu}\left[P_{\Delta}\left(\mathbf{1}_{A} Q_{k}\left(\mathbf{1}_{\mathbb{R}^{p}}\right)\right)\right] / \pi_{k-1}^{\mu}\left[P_{\Delta}\left(Q_{k}\left(\mathbf{1}_{\mathbb{R}^{p}}\right)\right)\right]$ have been used.

For the second claim, the fact that $\phi_{j, \infty}$ is log-concave for every $j \in \mathbb{N}_{0}$ follows from its definition as the pointwise limit in (3.4) and the log-concavity of $\varphi_{j, k}$ established in lemma 3. By lemma 2, $P_{\Delta} \phi_{k+1}$ is log-concave and since by assumption $2, \phi_{k, \infty}=\varsigma_{k}^{-1} Q_{k+1} \phi_{k+1, \infty}$, we may take $\tilde{\phi}_{k, \infty}(x)=$ $\varsigma_{k}^{-1} \tilde{g}_{k}\left(x, y_{k}\right) P_{\Delta} \phi_{k+1, \infty}(x)$.

Since $\pi_{k}^{\mu}$ has the interpretation of the conditional distribution of $x_{k \Delta}$ given $\left(y_{0}, \ldots, y_{k}\right)$, the measure $\pi_{k}^{\mu} \cdot\left(P_{\Delta} \varphi_{k+1, \ell}\right) / \pi_{k}^{\mu} P_{\Delta} \varphi_{k+1, \ell}$ is the so-called smoothing distribution which conditions additionally on $\left(y_{k+1}, \cdots, y_{k+\ell}\right)$. The interpretation of (3.4) is then that $\phi_{k, \infty}$ is the function with which to re-weight $\pi_{k}^{\mu} P_{\Delta}$ in order to condition on the infinite data record $\left(y_{k+\ell}\right)_{\ell \in \mathbb{N}_{0}}$. Indeed it is clear from (3.6) that assumption 2 implies that the filtering and smoothing measures, $\pi_{k}^{\mu}$ and $\pi_{k, \infty}^{\mu}$, are equivalent, despite the fact that $\pi_{k, \infty}^{\mu}$ conditions on an infinite number of observations.

The question of whether there exists a function which achieves this conditioning is itself closely connected to the question of filter stability. For a general class of discrete-time filtering problems with an ergodic signal and nondegenerate observations, it is shown in [28, Lemma 3.8] (see also the commentary immediately after the proof of Lemma 3.6 in the same article), that the transition kernel of the signal conditional on an infinite future data record is absolutely continuous w.r.t. to the (unconditional) transition kernel of the signal. In the notation of the present work this is, for each $k$, the absolute continuity of $R_{k, \infty}(x, \cdot)$ w.r.t. $P_{\Delta}(x, \cdot)$, i.e. $\phi_{k, \infty}$ is (a version of) the corresponding Radon-Nikodym derivative up to a factor depending on $x$. See [31] for a discussion on doubly infinite time horizons but under much more restrictive conditions. Assumption 2 requires such a derivative to not only exist but also satisfy certain regularity conditions, which below shall be verified in the setting of a specific example using the techniques of [30]. It is an open question whether assumption 2 can be deduced directly from theorem 1 .

When assumption 2 holds, we shall consider the family of weighted Wasserstein distances

$$
W_{q, k}(\mu, \nu):=W_{q}\left(\frac{\mu \cdot P_{\Delta} \phi_{k+1, \infty}}{\mu P_{\Delta} \phi_{k+1, \infty}}, \frac{\nu \cdot P_{\Delta} \phi_{k+1, \infty}}{\nu P_{\Delta} \phi_{k+1, \infty}}\right), \quad k \in \mathbb{N}_{0},
$$

whenever $\mu, \nu$ satisfy appropriate integrability conditions for these distances to be well-defined. The interest in the distances $W_{q, k}$ is due to the identity:

$$
W_{q, k}\left(\pi_{k}^{\mu}, \pi_{k}^{\nu}\right)=W_{q}\left(\pi_{k, \infty}^{\mu}, \pi_{k, \infty}^{\nu}\right),
$$

which follows from (3.6). Thus $W_{q, k}$ quantifies distance between $\pi_{k}^{\mu}$ and $\pi_{k}^{\nu}$ as the $W_{q}$-distance between the corresponding smoothing distributions $\pi_{k, \infty}^{\mu}$ and $\pi_{k, \infty}^{\nu}$.

We denote the set of probability measures

$$
\mathcal{P}_{q}:=\left\{\mu \text { on } \mathcal{B}\left(\mathbb{R}^{p}\right): \int\left(1+\|u\|^{q}\right) \phi_{0}(u) \mu(\mathrm{d} u)<\infty \quad \text { and } \quad \pi_{k}^{\mu} P_{\Delta} \phi_{k+1, \infty}<\infty, \forall k \in \mathbb{N}_{0}\right\}
$$


Theorem 2. If assumption 1 holds and for a given observation sequence $\left(y_{k}\right)_{k \in \mathbb{N}_{0}}$ assumption 2 holds, then for any $q \geq 1$,

$$
W_{q, k}\left(\pi_{k}\left(\mu, y_{0: k}, \cdot\right), \pi_{k}\left(\nu, y_{0: k}, \cdot\right)\right) \leq \exp \left[-\sum_{j=1}^{k} \int_{0}^{\Delta} \lambda\left(j, y_{j}, t\right) \mathrm{d} t\right] W_{q, 0}\left(\pi_{0}^{\mu}, \pi_{0}^{\nu}\right), \quad \forall k \geq 1, \mu, \nu \in \mathcal{P}_{q},
$$

where $\lambda\left(j, y_{j}, t\right)$ is as in theorem 1.

Given the identities (3.7) and (3.8), the proof of theorem 2 follows almost exactly the same programme as the proof of theorem 1, except working with the kernels $R_{k, \infty}$, the functions $\phi_{k, \infty}$ and their log-concavity in lemma 11, instead of $R_{j, k}, \varphi_{j, k}$ and their log-concavity in lemma 3 . Therefore the details are omitted. The requirement $\mu, \nu \in \mathcal{P}_{q}$ ensures that $W_{q, 0}(\mu, \nu)$ and $\pi_{k, \infty}^{\mu}, \pi_{k, \infty}^{\nu}$ are well-defined.

\section{Example: dynamic logistic regression}

As an example of the dynamic Generalized Linear Models described in section 2.5.3, consider the case: $\sigma^{2}>0, \beta$ such that $\lambda_{\text {sig }}>0$, and with $\mathbb{Y}=\{0,1\}^{n}$, the observations $Y_{k}=\left[Y_{k}^{(1)} \cdots Y_{k}^{(n)}\right]^{T}$ are conditionally independent given $x_{k \Delta}$, with the conditional probability of $\left\{Y_{k}^{i}=1\right\}$ being $1 /(1+$ $e^{-\sum_{j} x_{k \Delta}^{(j)} z_{k}^{(i, j)}}$ ), where $z_{k}^{(i, j)}$ are known covariates. The likelihood function at time $k$ is then:

$$
g_{k}\left(x, y_{k}\right)=\exp \left[\sum_{i=1}^{n}\left\{\sum_{j=1}^{p} y_{k}^{(i)} z_{k}^{(i, j)} x^{(j)}-\log \left(1+e^{\sum_{j=1}^{p} z_{k}^{(i, j)} x^{(j)}}\right)\right\}\right] .
$$

For any $\left(y_{k}\right)_{k \in \mathbb{N}_{0}}$, assumption 1 is satisfied with $\lambda_{g}\left(k, y_{k}\right)=0$, and therefore (2.17) holds by theorem 1. Checking assumption 2 is more involved, we shall use some results from [30].

Let us assume that the covariates satisfy

$$
\sup _{k \geq 0} \sum_{i, j}\left(z_{k}^{(i, j)}\right)^{2}<\infty
$$

and fix an arbitrarily sequence of observations $\left(y_{k}\right)_{k \in \mathbb{N}_{0}}$.

The following properties of this model are easily checked (see [30, Sec. 3.1] for a similar example): there exists a constant $c>0$ such that with

$$
V(x):=1+c\|x\|, \quad C_{d}:=\left\{x \in \mathbb{R}^{p}: V(x) \leq d\right\},
$$

we have for some $\underline{d} \in[1, \infty)$ and all $d \geq \underline{d}$,

- $\sup _{k} g_{k}\left(x, y_{k}\right) \leq 1, \forall x \in \mathbb{R}^{p}$, and there exist constants $\delta \in(0,1), b_{d} \in[0, \infty)$ such that

$$
P_{\Delta}\left(e^{V}\right) \leq \exp \left(V(1-\delta)+b_{d} \mathbf{1}_{C_{d}}\right)
$$

- $\inf _{k} g_{k}\left(x, y_{k}\right) P_{\Delta}\left(x, C_{d}\right)>0, \forall x \in \mathbb{R}^{p}$,

- there exist constants $\epsilon_{d}^{-}, \epsilon_{d}^{+}$such that $\forall x \in C_{d}$ and $k \in \mathbb{N}_{0}$,

$$
\epsilon_{d}^{-} \nu_{d}\left(\mathrm{~d} x^{\prime}\right) \mathbf{1}_{C_{d}}\left(x^{\prime}\right) \leq g_{k}\left(x, y_{k}\right) P_{\Delta}\left(x, \mathrm{~d} x^{\prime}\right) \mathbf{1}_{C_{d}}\left(x^{\prime}\right) \leq \epsilon_{d}^{-} \nu_{d}\left(\mathrm{~d} x^{\prime}\right) \mathbf{1}_{C_{d}}\left(x^{\prime}\right),
$$

where the probability measure $\nu_{d}$ is the normalized restriction of Lebesgue measure to $C_{d}$.

Define the norm on functions $f: \mathbb{R}^{p} \rightarrow \mathbb{R},\|f\|_{e^{V}}:=\sup _{x}|f(x)| / e^{V(x)}$. 
Proposition 3. For any $\mu_{0}$ such that $\mu_{0}\left(e^{V}\right)<\infty$, define $\phi_{j, k}(x):=\varphi_{j, k}(x) / \pi_{j-1}^{\mu_{0}} P_{\Delta} \varphi_{j, k}$. Then:

1) $\sup _{k \geq 0} \eta_{k}^{\mu_{0}}\left(e^{V}\right)<\infty$

2) $\sup _{0 \leq j \leq k}\left\|\phi_{j, k}\right\|_{e^{V}}<\infty$,

3) for all $d \geq \underline{d}, \inf _{0 \leq j \leq k} \inf _{x \in C_{d}} \phi_{j, k}(x)>0$,

4) for all $0<j \leq k, Q_{j} \phi_{j, k}=\varsigma_{j-1} \phi_{j-1, k}$, where $\varsigma_{j}=\int \eta_{j}^{\mu_{0}}(\mathrm{~d} x) g_{j}\left(x, y_{j}\right)$,

5) there exist constants $\rho<1$ and $c_{\mu_{0}}<\infty$ such that for any $f: \mathbb{R}^{p} \rightarrow \mathbb{R}$ with $\|f\|_{e^{V}}<\infty$,

$$
\left|\frac{Q_{j, k} f(x)}{\prod_{i=j}^{k-1} \varsigma_{i}}-\phi_{j, k-1}(x) \eta_{k}^{\mu_{0}} f\right| \leq \rho^{k-j}\|f\|_{e^{V}} c_{\mu_{0}} e^{V(x)} \mu_{0}\left(e^{V}\right), \quad \forall x \in \mathbb{R}^{p}, 0 \leq j<k
$$

Proof. The properties identified immediately before the statement of proposition and the requirement $\mu_{0}\left(e^{V}\right)<\infty$ imply that conditions (H1)-(H4) of [30] are satisfied. Then 1) and 2) are established by [30, Prop. 1 and 2], 3) by [30, Lem. 10], 4) by [30, Lem.1], and 5) by [30, Thm. 1].

The following proposition establishes that the conditions of theorem 2 are satisfied.

Proposition 4. For any sequence of observations $\left(y_{k}\right)_{k \in \mathbb{N}_{0}}$, the dynamic logistic regression model described above satisfies assumption 2 with $\sup _{k \geq 0}\left\|\phi_{k, \infty}\right\|_{e^{V}}<\infty$, and for any $q \geq 1$,

$$
W_{q, k}\left(\pi_{k}\left(\mu, y_{0: k}, \cdot\right), \pi_{k}\left(\nu, y_{0: k}, \cdot\right)\right) \leq \exp \left(-k \Delta \lambda_{\text {sig }}\right) W_{q, 0}\left(\pi_{0}^{\mu}, \pi_{0}^{\nu}\right)
$$

for all $\mu, \nu$ in the set of probability measures $\left\{\mu\right.$ on $\left.\mathcal{B}\left(\mathbb{R}^{p}\right): \int\left(1+\|x\|^{q}\right) e^{c\|x\|} \mu(\mathrm{d} x)<\infty\right\}$ where $c$ is as in (3.10).

Remark 1. The constant $\rho<1$ appearing in part 5) of proposition 3 and obtained using the techniques of [30] may degrade with dimension of the state-space. Note however, that $\rho$ does not appear in (3.12), it only serves as an intermediate tool used to in the following proof to help establish that assumption 2 holds.

Proof of proposition 4. Choose any $\mu_{0}$ such that $\mu_{0}\left(e^{V}\right)<\infty$. Noting the identities $\pi_{k-1}^{\mu_{0}} P_{\Delta} \varphi_{k, \ell}=$ $\prod_{j=k}^{\ell} \varsigma_{j}$ and $\phi_{j, k}=Q_{j, k+1} \mathbf{1}_{\mathbb{R}^{p}} / \prod_{i=j}^{k} \varsigma_{i}$, we have for any $\ell \geq 1$,

$$
\phi_{j, k}-\phi_{j, k+\ell}=\frac{Q_{j, k+1}}{\prod_{i=j}^{k} \varsigma_{i}}\left(1-\frac{Q_{k+1, k+\ell+1} \mathbf{1}_{\mathbb{R}^{p}}}{\prod_{i=k+1}^{k+\ell} \varsigma_{i}}\right) .
$$

Since $\prod_{i=k+1}^{k+\ell} \varsigma_{i}=\eta_{k+1}^{\mu_{0}} Q_{k+1, k+\ell+1} \mathbf{1}_{\mathbb{R}^{p}}$, we have $\eta_{k+1}^{\mu_{0}}\left(1-\frac{Q_{k+1, k+\ell+1} \mathbf{1}_{\mathbb{R} p}}{\prod_{i=k+1}^{k+\ell} \varsigma_{i}}\right)=0$ and by part 2$)$ of proposition $3, \sup _{j, k, \ell} \frac{\left\|Q_{k+1, k+\ell+1} \mathbf{1}_{\mathbb{R}} p\right\|_{e} V}{\prod_{i=k+1}^{k+\ell} \varsigma_{i}}=: c_{Q}<\infty$, so an application of part 5) of proposition 3 gives:

$$
\left\|\phi_{j, k}-\phi_{j, k+\ell}\right\|_{e^{V}} \leq \rho^{k+1-j} c_{Q} c_{\mu_{0}} \mu_{0}\left(e^{V}\right), \quad \forall \ell \geq 1 .
$$

It follows for each $j,\left(\phi_{j, k}\right)_{k \geq j}$ is a Cauchy sequence in the Banach space of functions $f: \mathbb{R}^{p} \rightarrow \mathbb{R}$ endowed with the norm $\|f\|_{e^{V}}<+\infty$. With the strong limit of $\left(\phi_{j, k}\right)_{k \geq j}$ then denoted $\phi_{j, \infty}$, we have $\left\|\phi_{j, \infty}\right\|_{e^{V}}<\infty$ and $\phi_{j, \infty}(x)=\lim _{k \rightarrow \infty} \phi_{j, k}(x)$ pointwise.

From part 4) of proposition 3 ,

$$
Q_{j} \phi_{j, k}=Q_{j} \phi_{j, \infty}+Q_{j}\left(\phi_{j, k}-\phi_{j, \infty}\right)=\varsigma_{j-1} \phi_{j-1, \infty}+\varsigma_{j-1}\left(\phi_{j-1, k}-\phi_{j-1, \infty}\right)=\varsigma_{j-1} \phi_{j-1, k},
$$

and since using (3.11), $\left\|Q_{j}\left(e^{V}\right)\right\|_{e^{V}}<\infty,\left\|\phi_{j-1, k}-\phi_{j-1, \infty}\right\| \rightarrow 0$ and $\left\|Q_{j}\left(\phi_{j, k}-\phi_{j, \infty}\right)\right\|_{e^{V}} \leq$ $\left\|Q_{j}\left(e^{V}\right)\right\|_{e^{V}}\left\|\phi_{j, k}-\phi_{j, \infty}\right\|_{e^{V}} \rightarrow 0$, both as $k \rightarrow \infty$, we have $Q_{j} \phi_{j, \infty}=\varsigma_{j-1} \phi_{j-1, \infty}$. Since $g_{j}\left(x, y_{j}\right) \in$ $(0,1)$, we have $\varsigma_{j} \in(0,1)$ and using part 3$)$ of proposition $3, Q_{j} \phi_{j, \infty}(x)>0$ for all $x$ hence $\phi_{j-1, \infty}(x)>$ 0 for all $x$. Also $\left\|\phi_{j, \infty}\right\|_{e^{V}}<\infty$ implies $\phi_{j, \infty}(x)<\infty$ for all $x$. The membership $\phi_{j-1, \infty} \in C^{2}$ follows from $Q_{j} \phi_{j, \infty}=\varsigma_{j-1} \phi_{j-1, \infty}$ together with $x \mapsto g_{j-1}\left(x, y_{j-1}\right) \in C^{2}$ by assumption 1 and the fact that $P_{\Delta}(x, \cdot)$ is Gaussian with mean depending linearly on $x$. That completes the verification of assumption 2 . 
To complete the proof, observe that in order for $\mu \in \mathcal{P}_{q}$ it is sufficient that $\int\left(1+\|x\|^{q}\right) e^{V(x)} \mu(\mathrm{d} x)<$ $\infty$, because using part 2) of proposition $3, \sup _{k \geq 0}\left\|\phi_{k, \infty}\right\|_{e^{V}}<\infty$, we have $\pi_{k-1}^{\mu} P_{\Delta}=\eta_{k}^{\mu}$ and by part 1) of proposition $3, \sup _{k} \eta_{k}^{\mu}\left(e^{V}\right)<\infty$.

Acknowledgement. The author thanks Anthony Lee for helpful comments.

\section{References}

[1] D. Bakry, I. Gentil, and M. Ledoux. Analysis and geometry of Markov diffusion operators, volume 348. Springer Science \& Business Media, 2013.

[2] P. Cattiaux and A. Guillin. Semi log-concave Markov diffusions. In Séminaire de probabilités XLVI, pages 231-292. Springer, 2014.

[3] D. Criśan and B. Rozovskii. The Oxford handbook of nonlinear filtering. Oxford University Press, 2011.

[4] Pierre Del Moral. Feynman-kac formulae. In Feynman-Kac Formulae, pages 47-93. Springer, 2004.

[5] Pierre Del Moral and Alice Guionnet. On the stability of measure valued processes with applications to filtering. Comptes Rendus de l'Académie des Sciences-Series I-Mathematics, 329(5):429434, 1999.

[6] C. Donner, K. Obermayer, and H. Shimazaki. Approximate inference for time-varying interactions and macroscopic dynamics of neural populations. PLoS computational biology, 13(1):1-27, 2017.

[7] R. Douc, G. Fort, É. Moulines, and P. Priouret. Forgetting the initial distribution for hidden Markov models. Stochastic processes and their applications, 119(4):1235-1256, 2009.

[8] R. Douc, E. Gassiat, B. Landelle, and É Moulines. Forgetting of the initial distribution for nonergodic hidden Markov chains. The Annals of Applied Probability, 20(5):1638-1662, 2010.

[9] R. Douc, É. Moulines, and D. Stoffer. Nonlinear time series: Theory, methods and applications with $R$ examples. Chapman and Hall/CRC, 2014.

[10] D.C. Dowson and B.V. Landau. The Fréchet distance between multivariate normal distributions. Journal of multivariate analysis, 12(3):450-455, 1982.

[11] M. Gerber and N. Whiteley. Stability with respect to initial conditions in V-norm for nonlinear filters with ergodic observations. Journal of Applied Probability, 54(1):118-133, 2017.

[12] J. Harrison and M. West. Bayesian forecasting $\& 3$ dynamic models, volume 1030 of Springer Series in Statistics. Springer New York City, 1999.

[13] E. Jacquier, N.G. Polson, and P.E. Rossi. Bayesian analysis of stochastic volatility models. Journal of Business \& Economic Statistics, 20(1):69-87, 2002.

[14] S. Kim, N. Shephard, and S. Chib. Stochastic volatility: likelihood inference and comparison with arch models. The review of economic studies, 65(3):361-393, 1998.

[15] M.L. Kleptsyna and A.Y. Veretennikov. On discrete time ergodic filters with wrong initial data. Probability Theory and Related Fields, 141(3-4):411-444, 2008.

[16] A.V. Kolesnikov. On diffusion semigroups preserving the log-concavity. Journal of Functional Analysis, 186(1):196-205, 2001. 
[17] P. McCullagh and J.A Nelder. Generalized Linear Models, volume 37 of Monograph on Statistics and Applied Probability. Chapman \& Hall,, 1989.

[18] K. P. Murphy. Machine Learning: A Probabilistic Perspective. MIT Press, 2012.

[19] Y. Nesterov. Lectures on convex optimization, volume 137. Springer, 2018.

[20] M. K. Pitt and N. Shephard. Time varying covariances: a factor stochastic volatility approach. Bayesian statistics, 6:547-570, 1999.

[21] A. Prékopa. On logarithmic concave measures and functions. Acta Scientiarum Mathematicarum, 34:334-343, 1973.

[22] P. Rebeschini and R. Van Handel. Can local particle filters beat the curse of dimensionality? The Annals of Applied Probability, 25(5):2809-2866, 2015.

[23] D. Revuz and M. Yor. Continuous martingales and Brownian motion. Springer, 3rd edition, 1999.

[24] H. Shimazaki, S. Amari, E. N. Brown, and S. Grün. State-space analysis of time-varying higher-order spike correlation for multiple neural spike train data. PLoS Computational Biology, 8:e1002385, 2012.

[25] W. Stannat. Stability of the Optimal Filter for Nonergodic Signals - A Variational Approach. In D. Criśan and B. Rozovskii, editors, The Oxford handbook of nonlinear filtering, chapter 13. Oxford University Press, 2011.

[26] R. Sundberg. Statistical modelling by exponential families, volume 12. Cambridge University Press, 2019.

[27] X.T. Tong and R. van Handel. Conditional ergodicity in infinite dimension. The Annals of Probability, 42(6):2243-2313, 2014.

[28] R. Van Handel. The stability of conditional Markov processes and markov chains in random environments. The Annals of Probability, 37(5):1876-1925, 2009.

[29] R. Van Handel. Nonlinear filtering and systems theory. In Proceedings of the 19th International Symposium on Mathematical Theory of Networks and Systems (MTNS semi-plenary paper), 2010.

[30] N. Whiteley. Stability properties of some particle filters. The Annals of Applied Probability, 23(6):2500-2537, 2013.

[31] N. Whiteley and A. Lee. Twisted particle filters. The Annals of Statistics, 42(1):115-141, 2014. 